\title{
Can serum albumin level affect the pharmacological action of tolvaptan in patients with liver cirrhosis? A post hoc analysis of previous clinical trials in Japan
}

\author{
Isao Sakaida $\cdot$ Koji Nakajima $\cdot$ Kiwamu Okita $\cdot$ Masatsugu Hori $\cdot$ \\ Tohru Izumi · Masaya Sakurai · Yoshiyuki Shibasaki · Sayaka Tachikawa • \\ Hidetsugu Tsubouchi $\cdot$ Hiromi Oka $\cdot$ Hiroyuki Kobayashi
}

Received: 12 January 2015/Accepted: 3 February 2015/Published online: 18 February 2015

(C) The Author(s) 2015. This article is published with open access at Springerlink.com

\begin{abstract}
Background Patients with hypoalbuminemia often fail to respond to increased doses of loop diuretics. We therefore performed a post hoc analysis to investigate the pharmacological action of tolvaptan and whether it is dependent on the serum albumin level.

Methods This analysis was based on four previous clinical trials of tolvaptan in patients with liver cirrhosis who exhibited insufficient response to conventional diuretics. We analyzed the correlation between the change in the initial 24-h cumulative urine volume from baseline and the serum albumin level at baseline, and assessed potential predictive factors of response to tolvaptan.
\end{abstract}

\section{Sakaida}

Department of Gastroenterology and Hepatology, Yamaguchi

University Graduate School of Medicine, Minami Kogushi

1-1-1, Ube, Yamaguchi 755-8505, Japan

e-mail: sakaida@yamaguchi-u.ac.jp

K. Nakajima ( $₫) \cdot$ M. Sakurai - Y. Shibasaki - S. Tachikawa · H. Tsubouchi $\cdot$ H. Oka $\cdot$ H. Kobayashi

Department of Medical Affairs, Otsuka Pharmaceutical Co. Ltd.,

Tokyo, Japan

e-mail: nakajimak@otsuka.jp

K. Okita

Shunan Memorial Hospital, Kudamatsu, Yamaguchi, Japan

\section{Hori}

Osaka Medical Center for Cancer and Cardiovascular Diseases

President Emeritus, Osaka, Japan

T. Izumi

Kitasato University, Sagamihara, Kanagawa, Japan

T. Izumi

Kojinkai Niigata Minami Hospital, Niigata, Japan
Results The correlation coefficient was 0.029 in the placebo group and -0.112 in the $7.5 \mathrm{mg}$ tolvaptan group of patients with liver cirrhosis. Administration of tolvaptan provoked a stable response regardless of the serum albumin level. Tolvaptan use was identified as a significant predictor of pharmacological action, and was shown to change the initial urine volume by $885 \mathrm{~mL}(P<0.0001)$ in liver cirrhosis patients.

Conclusions In this post hoc analysis, tolvaptan increased the initial urine volume from baseline regardless of serum albumin levels. Use of tolvaptan as an add-on therapy to loop diuretics can be considered an optimal therapeutic option in patients with insufficient response to loop diuretics.

Keywords Tolvaptan - Initial urine volume - Albumin · Diuretics · Liver cirrhosis

\section{Introduction}

Volume overload is a phenomenon associated with liver cirrhosis, and its mainstay treatment is loop diuretics. The loop diuretic dosage is increased until diuresis is satisfactory $[1,2]$. However, if diuresis remains unsatisfactory, few useful alternative therapeutic options are available for patients with volume overload. In addition, it is well known that increases in loop diuretic dosage are not well tolerated and can lead to adverse events, including renal impairment and electrolyte abnormality [3, 4].

Hypoalbuminemia frequently complicates liver cirrhosis [5]. The serum albumin level contributes to the prevention of edema by balancing the hydrostatic and colloid osmotic pressure within blood vessels $[6,7]$. The binding of drugs to serum albumin plays an important role in pharmacokinetics, drug distribution, and drug metabolism 
$[6,8]$. Hypoalbuminemia is considered a reason for insufficient response to loop diuretics [9]. Few therapeutic options are available for patients with hypoalbuminemia if the response to loop diuretics is insufficient [10].

Tolvaptan is a novel diuretic agent that acts on the distal nephron to allow excretion of electrolyte-free water (aquaresis), thereby exerting its pharmacological action without causing electrolyte imbalance [11]. In the four previous clinical trials, tolvaptan was shown to reduce body weight in patients with liver cirrhosis [12-15] who had insufficient response to conventional diuretics. Change in body weight was considered a reliable surrogate marker of improvement in symptoms in several previous clinical trials of diuretic therapy [12-18]. Short-term outcomes of diuretic therapy include an increased urine volume and a reduced body weight. We therefore considered that change in the initial 24-h cumulative urine volume (change in the initial urine volume) from baseline might be an appropriate measure of the diuretic effect and might be assessable during short-term treatment. Furthermore, previous studies on tolvaptan did not evaluate whether the change in the initial urine volume from baseline is related to the serum albumin level at baseline [12-15].

In a study of tolvaptan efficacy in patients with liver cirrhosis, Sakaida et al. [15] reported a significant reduction in body weight after tolvaptan treatment compared with treatment with placebo despite a serum albumin level of less than $2.5 \mathrm{~g} / \mathrm{dL}$. However, no study has assessed whether the tolvaptan-induced change in the initial urine volume depends on the serum albumin level at baseline. Therefore, we performed a post hoc analysis to investigate whether the change in the initial urine volume from baseline is related to the serum albumin level at baseline. In addition, factors affecting the change in the initial urine volume were analyzed.

\section{Methods}

\section{Objectives}

The primary objective of this post hoc analysis was to evaluate the correlation between the change in the initial urine volume from baseline and the serum albumin level at baseline in patients with liver cirrhosis who were treated with tolvaptan or placebo. The secondary objective was to identify the most reliable factors predictive of the change in the initial urine volume.

\section{Study population}

This post hoc analysis was based on four previous clinical trials of tolvaptan performed in patients with liver cirrhosis
[12-15] in Japan to obtain indication for use. Patients in these trials received loop diuretic therapy before use of the study drugs. Pharmacokinetic and pharmacodynamic, escalating dose, dose finding, and pivotal trials were conducted to investigate, respectively, pharmacokinetics and pharmacodynamics [12], safety and efficacy of seven or more consecutive days of treatment after escalation of the dose [13], placebo-controlled dose response [14], and efficacy and safety of treatment over seven consecutive days [15] in patients with liver cirrhosis. The designs of these trials have been detailed [12-15].

Eligible patients with liver cirrhosis included those aged 20-80 years with persistent ascites despite combination diuretic therapy (a loop diuretic and an antialdosterone agent) before the start of the study. Patients with a total bilirubin level greater than $4.0 \mathrm{mg} / \mathrm{dL}$, serum creatinine level greater than $2.0 \mathrm{mg} / \mathrm{dL}$, serum sodium level greater than $147 \mathrm{mEq} / \mathrm{L}$, and/or serum potassium level greater than $5.5 \mathrm{mEq} / \mathrm{L}$ as well as patients who received an albumin infusion within 7 days before starting the trial were excluded. Albumin infusion was not allowed during the treatment period.

These trials were conducted in accordance with the ethical principles stated in the Declaration of Helsinki [19] and in compliance with good clinical practice guidelines [20]. The protocols were approved by the institutional review board at each trial site. All patients provided written informed consent.

\section{Study design}

Inclusion and exclusion criteria of these trials were similar for all liver cirrhosis patients, and the present post hoc analysis was performed on the pooled data from the four trials.

Data from patients with liver cirrhosis who had received $7.5 \mathrm{mg}$ tolvaptan once daily ( $7.5 \mathrm{mg}$ tolvaptan group) or placebo (placebo group) were extracted for the post hoc analysis. The full analysis set included all randomized patients who received trial drugs at least once. Among the selected patients, those with a missing baseline serum albumin level, or baseline or initial urine volume were excluded from the analysis.

The baseline was defined as a 3-day observation period before the administration of the study drugs. The initial urine volume was defined as the urine volume collected during the first $24 \mathrm{~h}$ after initiation of treatment with the study drugs. The baseline urine volume was defined as the 24-h cumulative urine volume measured during the 3-day observation period (baseline). Changes in the initial urine volume from baseline were compared between the $7.5 \mathrm{mg}$ tolvaptan and placebo groups. Moreover, an analysis of the change in the initial urine volume from baseline was 
conducted after stratification of patients into a subgroup with a serum albumin level below $2.5 \mathrm{~g} / \mathrm{dL}$ and a subgroup with a serum albumin level of $2.5 \mathrm{~g} / \mathrm{dL}$ or greater.

Factors affecting the change in the initial urine volume from baseline-including the study drug (tolvaptan), serum albumin level, age, sex, body weight, urine volume, serum creatinine level, serum sodium level, blood urea nitrogen (BUN) level, alanine aminotransferase level, aspartate aminotransferase level, total bilirubin level, and loop diuretic dose-were selected and assessed in all four clinical trials of tolvaptan. Previously, baseline renal function parameters, including serum albumin level and electrolyte levels, appeared to predict the change in the initial urine volume from baseline when loop diuretics were administered [21].

\section{Statistical analysis}

Baseline characteristics and demographic data of patients with liver cirrhosis were analyzed in the $7.5 \mathrm{mg}$ tolvaptan and placebo groups. Continuous data were expressed as the mean \pm the standard deviation, and were analyzed using Student's $t$ test. Categorical data were expressed as a number or percentage, and were analyzed using Fisher's exact test. The correlation between the change in the initial urine volume from baseline and the serum albumin level at baseline was examined by linear regression analysis. The Pearson correlation coefficient $(r)$ was used to assess this correlation.

Potential predictors of the change in the initial urine volume were investigated by multiple regression analysis. The data were expressed as an estimate, the standard error, the $95 \%$ confidence interval $(\mathrm{CI})$, and the $P$ value. An estimate was defined as the predicted change in the initial urine volume depending on the baseline data. A two-tailed $P$ value below 0.05 was considered statistically significant. All statistical analyses were performed using SAS 9.4 (SAS Institute, Cary, NC, USA) and JMP version 11.0.0 (SAS Institute, Cary, NC, USA).

\section{Results}

\section{Patients}

The number of enrolled patients and the analysis set for the present post hoc analysis are shown in Fig. 1. Of the 284 patients, 106 were enrolled in the placebo group and 178 were enrolled in the $7.5 \mathrm{mg}$ tolvaptan group; five patients [three patients $(2.8 \%)$ in the placebo group and two patients $(1.1 \%)$ in the $7.5 \mathrm{mg}$ tolvaptan group, respectively] were excluded because of missing data, and thus the data on 103 patients in the placebo group and on
Liver cirrhosis

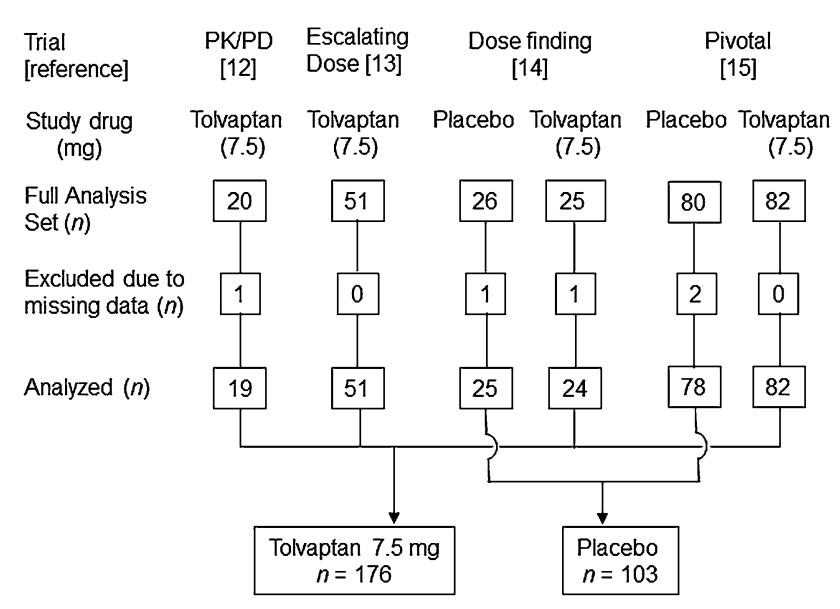

Fig. 1 Enrolled patients and analysis set. The data are expressed as the number of patients. Numbers in square brackets represent reference citations. $P K / P D$ pharmacokinetics/pharmacodynamics

176 patients in the $7.5 \mathrm{mg}$ tolvaptan group were analyzed. Characteristics and demographic data of the patients are shown in Table 1 . No significant differences were observed in any characteristic between the placebo and $7.5 \mathrm{mg}$ tolvaptan groups.

\section{Efficacy outcomes}

The change in the initial urine volume was significantly greater in the $7.5 \mathrm{mg}$ tolvaptan group than in the placebo group $(104 \pm 549 \mathrm{~mL}$ vs $1,026 \pm 739 \mathrm{~mL}, P<0.0001)$.

There was no correlation between the change in the initial urine volume from baseline and the serum albumin level at baseline ( $r=0.029$ in the placebo group and $r=-0.112$ in the $7.5 \mathrm{mg}$ tolvaptan group; Fig. 3a), as shown in Fig. 3 . Tolvaptan (compared with placebo) was associated with a significant change in the initial urine volume in patients with a serum albumin level below $2.5 \mathrm{~g} / \mathrm{dL}(32 \pm 526 \mathrm{~mL}$ vs $1,079 \pm 653 \mathrm{~mL}, P<0.0001)$, as well as in patients with a serum albumin level of $2.5 \mathrm{~g} / \mathrm{dL}$ or greater $(123 \pm 556 \mathrm{~mL}$ vs $1,005 \pm 772 \mathrm{~mL}, P<0.0001$; Fig. $3 \mathrm{~b}$ ).

Results of the analysis of predictive factors are shown in Table 2. Tolvaptan use, age, and serum BUN level were identified as predictive factors. Tolvaptan use was associated with an estimated $885-\mathrm{mL}$ change in the initial urine volume (95\% CI $727-1,043 \mathrm{~mL}, P<0.0001)$. A 1 -year increase in age was associated with an estimated $-11 \mathrm{~mL}$ change in the initial urine volume $(95 \% \mathrm{CI}-20$ to $-2 \mathrm{~mL}, P=0.0167)$. An increase in the serum BUN level per unit was associated with an estimated $-12-\mathrm{mL}$ change in the initial urine volume $(95 \% \mathrm{CI}-24$ to $-1 \mathrm{~mL}$, $P=0.0355$ ). The serum albumin level at baseline was not a significant predictive factor. 
Table 1 Demographic and baseline characteristics of patients with liver cirrhosis $(n=279)$

\begin{tabular}{llll}
\hline & Tolvaptan $(n=176)$ & Placebo $(n=103)$ & $P$ \\
\hline Male sex & $118(67.0 \%)$ & $64(62.1 \%)$ & 0.4358 \\
Age (years) & $65.01 \pm 9.24$ & $65.83 \pm 9.17$ & 0.4742 \\
Body weight $(\mathrm{kg})$ & $60.32 \pm 11.67$ & $58.65 \pm 12.90$ & 0.2685 \\
Urine volume $(\mathrm{mL})$ & $1,473.56 \pm 681.78$ & $1,394.78 \pm 565.55$ & 0.3230 \\
BUN (mg/dL) & $23.39 \pm 11.23$ & $24.03 \pm 11.62$ & 0.6500 \\
Serum creatinine (mg/dL) & $1.01 \pm 0.36$ & $1.01 \pm 0.41$ & 0.9767 \\
Serum albumin (g/dL) & $2.81 \pm 0.48$ & $2.85 \pm 0.50$ & 0.5135 \\
Serum sodium (mEq/L) & $135.13 \pm 4.58$ & $135.69 \pm 4.18$ & 0.3063 \\
ALT (IU/L) & $28.07 \pm 18.43$ & $27.73 \pm 22.26$ & 0.8907 \\
AST (IU/L) & $49.97 \pm 30.17$ & $47.71 \pm 38.45$ & 0.5861 \\
T-Bil (mg/dL) & $1.56 \pm 0.88$ & $1.46 \pm 0.97$ & 0.3741 \\
Loop diuretic dose (mg) & $64.32 \pm 34.49$ & $61.17 \pm 27.98$ & 0.4312 \\
\hline
\end{tabular}

Data are expressed as the number and percentage or the mean \pm standard deviation. Comparisons between the $7.5 \mathrm{mg}$ tolvaptan and placebo groups were performed using Student's $t$ test for continuous data and Fisher's exact test for categorical data. The loop diuretic dose was converted to the equivalent dose of furosemide.

$A L T$ alanine aminotransferase, $A S T$ aspartate aminotransferase, $B U N$ blood urea nitrogen, $T$-Bil total bilirubin

Table 2 Factors predictive of the pharmacological action of tolvaptan in patients with liver cirrhosis $(n=279)$

\begin{tabular}{lrrrr}
\hline Predictive factor & Estimate & SE & $P$ & $95 \%$ CI \\
\hline Intercept & -40.0 & $1,349.55$ & 0.9764 & $-2,697.2$ to $2,617.3$ \\
Study drug (tolvaptan use) & 885.0 & 80.12 & $<0.0001$ & 727.2 to $1,042.7$ \\
Serum albumin level & 20.7 & 87.12 & 0.8120 & -150.8 to 192.3 \\
Age & -11.0 & 4.58 & 0.0167 & -20.0 to -2.0 \\
Sex (male) & 157.2 & 96.41 & 0.1041 & -32.6 to 347.1 \\
Body weight & 0.2 & 4.13 & 0.9528 & -7.9 to 8.4 \\
Urine volume & -0.0 & 0.07 & 0.5640 & -0.2 to 0.1 \\
Serum creatinine level & -45.8 & 171.76 & 0.7900 & -384.0 to 292.4 \\
Serum sodium level & 5.8 & 9.39 & 0.5369 & -12.7 to 24.3 \\
BUN level & -12.2 & 5.78 & 0.0355 & -23.6 to -0.8 \\
ALT level & -1.6 & 3.82 & 0.6675 & -9.2 to 5.9 \\
AST level & 2.1 & 2.36 & 0.3746 & -2.5 to 6.7 \\
T-Bil level & 82.5 & 50.82 & 0.1058 & -17.6 to 182.6 \\
Loop diuretic dose & 2.1 & 1.24 & 0.0914 & -0.3 to 4.5 \\
\hline
\end{tabular}

Factors predictive of the change in the initial urine volume were investigated by multiple regression analysis. Data are expressed as an estimate, the standard error $(S E)$, the $95 \%$ confidence interval $(C I)$, and the $P$ value. The loop diuretic dose was converted to the equivalent dose of furosemide.

$A L T$ alanine aminotransferase, $A S T$ aspartate aminotransferase, BUN blood urea nitrogen, SE standard error, $T$-Bil total bilirubin

\section{Discussion}

In the present post hoc analysis, administration of tolvaptan increased the initial urine volume from baseline by approximately $1,000 \mathrm{~mL}$ in patients with liver cirrhosis and insufficient response to conventional diuretics (Fig. 2). This pharmacological action of tolvaptan was independent of the baseline serum albumin level. No change in the initial urine volume was observed in the placebo group. Our short-term study found no increase in urine volume without tolvaptan supplementation of the ongoing diuretic regimens and no correlation between the change in the initial urine volume and the serum albumin level in patients with liver cirrhosis.

We hypothesized that certain factors predict the pharmacological action of tolvaptan. Additional analysis regarding the relationship between potential predictive factors and the change in the initial urine volume showed that tolvaptan use was the most reliable factor predicting the change in the initial urine volume. This is the first study 
to use statistical methods to identify the most reliable factor predictive of a change in the initial urine volume in patients with liver cirrhosis. Tolvaptan use but not the serum albumin level at baseline was estimated to have a strong relationship with the change in the initial urine volume. Tolvaptan add-on therapy was effective in liver cirrhosis patients with a low serum albumin level (below $2.5 \mathrm{~g} / \mathrm{dL}$ ) and in liver cirrhosis patients with a high serum albumin level (2.5 g/dL or greater) (Fig. 3b) [15]. Other factors-namely, age and serum BUN level-showed a weak relationship with the change in the initial urine volume in patients with liver cirrhosis. We believe it is

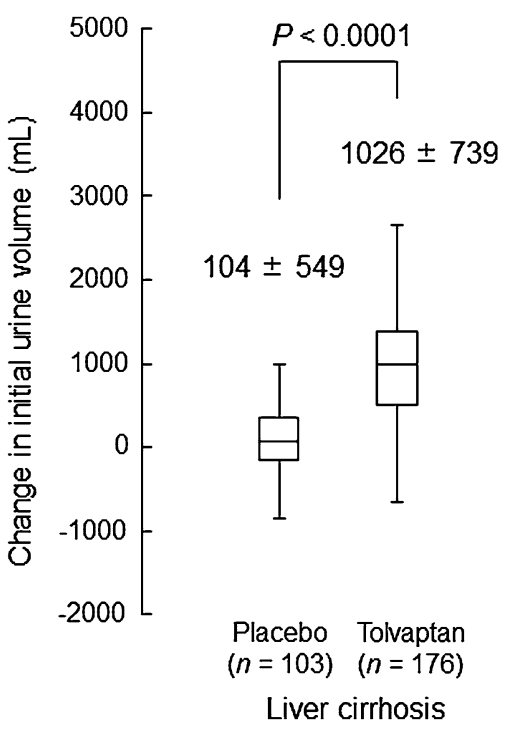

Fig. 2 The change in the initial urine volume in response to treatment (tolvaptan vs placebo) is compared in patients with liver cirrhosis. The data are expressed as the mean \pm the standard deviation. Statistical analysis was performed using Student's $t$ test reasonable that older patients may have a lesser increase or a decrease in urine volume, as our data suggest. However, at this juncture we do not fully understand why a higher BUN level would correlate with less urine, especially when there is no obvious correlation between urine volume and serum creatinine level. We suggest the correlation between a higher BUN level and less urine in the presence of normal levels of serum creatinine may reflect dehydration in patients with already altered liver function.

It is well known that loop diuretics are not sufficiently effective in patients with hypoalbuminemia [22-24]. In the present post hoc analysis, tolvaptan added to treatment with conventional diuretics significantly increased the initial urine volume compared with placebo, even in patients with low serum albumin levels. In a previous seminal study, administration of furosemide-albumin complex efficiently promoted diuresis by increasing urine volume in patients with hypoalbuminemia [25]. In addition, a randomized clinical trial in patients with liver cirrhosis indicated the potent effect of co-administration of furosemide and albumin on ascites resolution and rehospitalization [26]. Furthermore, a recent randomized controlled study in patients with hypoalbuminemic chronic kidney disease showed superior short-term efficacy of the combination of furosemide and albumin over furosemide alone in enhancing water and sodium diuresis [21].

The albumin-bound fraction of a loop diuretic reaches the proximal tubular epithelial cells to interact with an anion transporter. It is then translocated into the tubular lumen to exert the diuretic effect in the ascending limb of Henle's loop [5]. However, hypoalbuminemia is theorized to contribute to diuretic resistance by increasing the volume of distributed protein-bound loop diuretics, thereby decreasing the amount of drug delivered to the renal

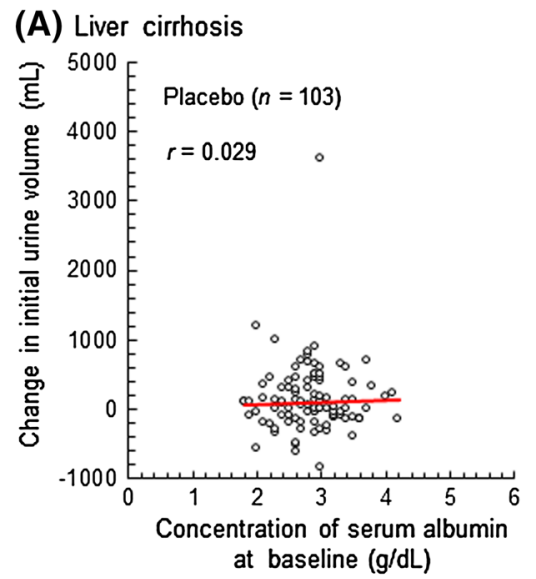

Fig. 3 Scatter plots (a) and box plots (b) examining the relationship between the change in the initial urine volume from baseline and the serum albumin level at baseline in patients with liver cirrhosis. The data in $\mathbf{a}$ are Pearson product-moment correlation coefficients
(B) Liver cirrhosis
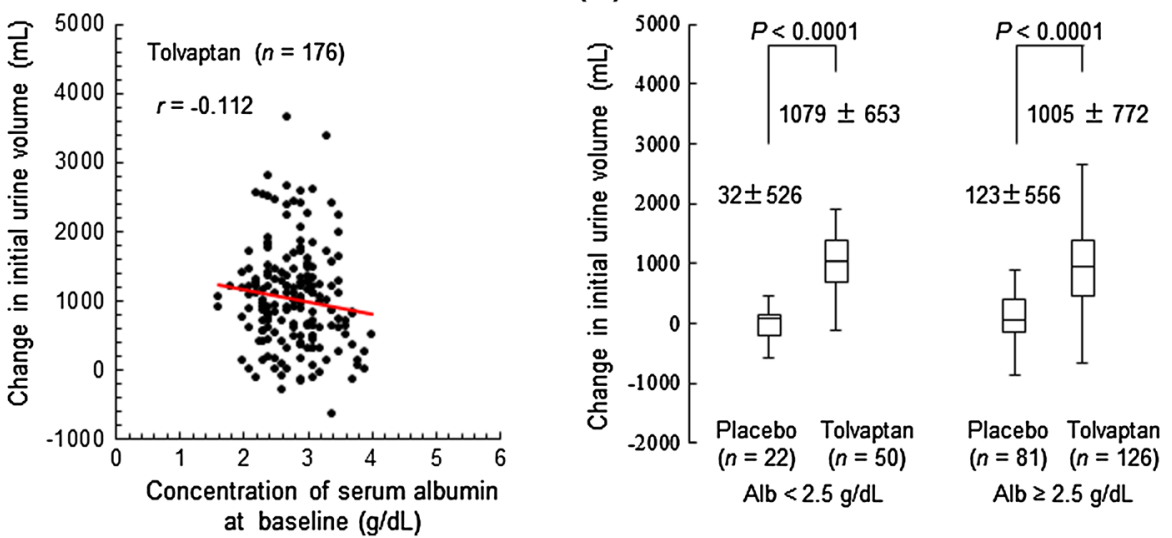

( $r$ ) obtained by linear regression analysis. The data in $\mathbf{b}$ are expressed as the mean \pm the standard deviation and were analyzed after stratification based on a serum albumin level of $2.5 \mathrm{mg} / \mathrm{dL}$. Statistical analysis was performed using Student's $t$ test. Alb albumin 
Fig. 4 Suggestion of novel diuretic therapy based on two different mechanisms of diuretic action. Tolvaptan has a mechanism of action different from that of loop diuretics, and therefore tolvaptan add-on therapy is expected to be an optimal therapeutic option in patients with insufficient response to loop diuretics. $A l b$ albumin, $A Q P$ aquaporin, $A V P$ arginine vasopressin, Fur furosemide, $O A T$ organic anion transporter, Tol tolvaptan

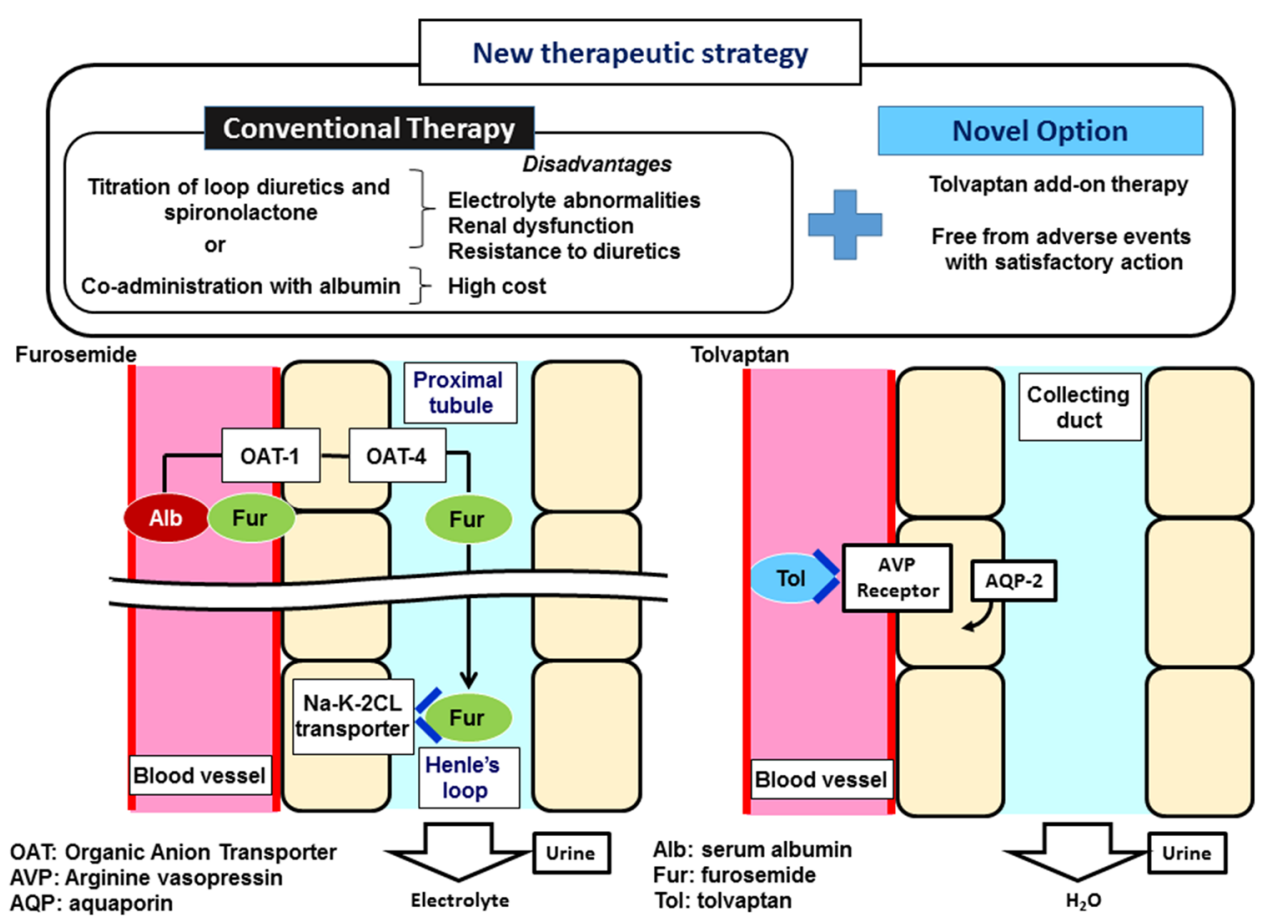

secretory sites [27]. Namely, the volume of distributed furosemide increases in hypoalbuminemic patients because the drug's retention time in plasma is short, leading to a decline in the amount of albumin-bound furosemide transferred to the proximal tubules. As a result, the level of albumin-free furosemide might be increased in the plasma of hypoalbuminemic patients. To some degree, the diuretic effect of furosemide is exerted in such patients by an increase in the dose. However, it is not clear whether albumin-free furosemide (in contrast to albumin-bound furosemide) has the potential to exert a diuretic effect via its transport to the ascending limb of Henle's loop. Thus, the diuretic effect of furosemide alone may be insufficient, because serum albumin levels are often low in patients with volume overload [28].

Investigation of the in vitro binding of $\left[{ }^{14} \mathrm{C}\right]$ tolvaptan to protein at concentrations of $0.1-10 \mu \mathrm{g} / \mathrm{mL}$ in mouse, rat, rabbit, dog, and human plasma found high binding values of $97.2 \%$ or greater in animals and $98.0-98.5 \%$ in humans [29]. Thus, both tolvaptan and furosemide have similarly high protein binding values. However, tolvaptan had a diuretic effect in patients with liver cirrhosis in the present post hoc analysis despite their having low serum albumin levels.

In patients with volume overload, release of vasopressin is increased in blood vessels [30]. The mechanism of tolvaptan involves antagonizing $\mathrm{V}_{2}$ receptors in such patients (Fig. 4) [11], and this difference in the mechanism of action between furosemide and tolvaptan is assumed to account for the protein-binding independence of tolvaptan's diuretic effect. Unfortunately, no evidence shows how much of the protein-free tolvaptan binds to $\mathrm{V}_{2}$ receptors in blood vessels and the extent to which protein-free tolvaptan binding to $\mathrm{V}_{2}$ receptors depends on serum albumin levels. Further examination of these issues is warranted.

Previous clinical trials showed that the most common adverse event associated with tolvaptan was an increase in thirst, and only a few serious adverse events were observed. Hypernatremia was observed during the postmarketing period in some patients with heart failure treated with tolvaptan [31]. Tolvaptan should be administered to patients with volume overload while they are receiving careful monitoring for hypernatremia.

In conclusion, this post hoc analysis shows that tolvaptan significantly increases the initial urine volume independent of serum albumin levels. Tolvaptan add-on therapy could be an optimal therapeutic option in patients with an insufficient response to loop diuretics.

Acknowledgments The data analyzed in the present post hoc analysis were all provided by the Department of Clinical Development, Otsuka Pharmaceutical, to the Department of Medical Affairs, Otsuka Pharmaceutical. Koji Nakajima contributed to the concept and design of the present analysis, data acquisition, data analysis, and interpretation of the data, wrote the first draft of the manuscript, critically revised the manuscript, and gave final approval for the submitted manuscript. Sayaka Tachikawa, Masaya Sakurai, and Yoshiyuki Shibasaki contributed to data analysis and interpretation of the data, take responsibility for the integrity of the data and accuracy of the data analysis, critically revised the manuscript; and gave final approval for the submitted manuscript. Hidetsugu Tsubouchi contributed to interpretation of the data, critically revised the manuscript, and gave final approval for the submitted manuscript. Isao Sakaida, 
Kiwamu Okita, Tohru Izumi, Masatsugu Hori, Hiromi Oka, and Hiroyuki Kobayashi critically revised the manuscript and gave final approval for the submitted manuscript.

Conflict of interest Isao Sakaida is a consultant for Otsuka Pharmaceutical and received research Grants from Otsuka Pharmaceutical, Takeda Pharmaceutical, MSD, and Chugai Pharmaceutical. Kiwamu Okita is a consultant for Otsuka Pharmaceutical. Masatsugu Hori is a consultant for Otsuka Pharmaceutical. Tohru Izumi is a consultant for Otsuka Pharmaceutical. Koji Nakajima, Masaya Sakurai, Yoshiyuki Shibasaki, Sayaka Tachikawa, Hidetsugu Tsubouchi, Hiromi Oka, and Hiroyuki Kobayashi are employees of Otsuka Pharmaceutical.

Open Access This article is distributed under the terms of the Creative Commons Attribution Noncommercial License which permits any noncommercial use, distribution, and reproduction in any medium, provided the original author(s) and the source are credited.

\section{References}

1. Biecker E. Diagnosis and therapy of ascites in liver cirrhosis. World J Gastroenterol. 2011;17:1237-48.

2. Hasselblad V, Gattis Stough W, Shah MR, et al. Relation between dose of loop diuretics and outcomes in a heart failure population: results of the ESCAPE trial. Eur J Heart Fail. 2007;9:1064-9.

3. Costanzo MR, Jessup M. Treatment of congestion in heart failure with diuretics and extracorporeal therapies: effects on symptoms, renal function, and prognosis. Heart Fail Rev. 2012;17:313-24.

4. Madan K, Mehta A. Management of renal failure and ascites in patients with cirrhosis. Int J Hepatol. 2011. doi:10.4061/2011/ 790232.

5. Kitsios GD, Mascari P, Ettunsi R, et al. Co-administration of furosemide with albumin for overcoming diuretic resistance in patients with hypoalbuminemia: a meta-analysis. J Crit Care. 2014;29:253-9.

6. Barchel D, Almoznino-Sarafian D, Shteinshnaider M, et al. Hypoalbuminemia. Intern Emerg Med. 2012;7(Suppl 3):S193-9.

7. Fauchald P. Transcapillary colloid osmotic gradient and body fluid volumes in renal failure. Kidney Int. 1986;29:895-900.

8. Nicholson JP, Wolmarans MR, Park GR. The role of albumin in critical illness. Br J Anaesth. 2000;85:599-610.

9. Tanzi M, Gardner M, Megellas M, et al. Evaluation of the appropriate use of albumin in adult and pediatric patients. Am J Health Syst Pharm. 2003;60:1330-5.

10. Narahara Y, Kanazawa H, Fukuda T, et al. Transjugular intrahepatic portosystemic shunt versus paracentesis plus albumin in patients with refractory ascites who have good hepatic and renal function: a prospective randomized trial. J Gastroenterol. 2011; 46:78-85.

11. Yamamura Y, Nakamura S, Itoh S, et al. OPC-41061, a highly potent human vasopressin V2-receptor antagonist: pharmacological profile and aquaretic effect by single and multiple oral dosing in rats. J Pharmacol Exp Ther. 1998;287:860-7.

12. Sakaida I, Yanase M, Kobayashi Y, et al. The pharmacokinetics and pharmacodynamics of tolvaptan in patients with liver cirrhosis with insufficient response to conventional diuretics: a multicentre, double-blind, parallel-group, phase III study. J Int Med Res. 2012;40:2381-93.
13. Sakaida I, Yamashita S, Kobayashi T, et al. Efficacy and safety of a 14-day administration of tolvaptan in the treatment of patients with ascites in hepatic oedema. J Int Med Res. 2013;41:835-47.

14. Okita K, Kawazoe S, Hasebe C, et al. Dose-finding trial of tolvaptan in liver cirrhosis patients with hepatic edema: a randomized, double-blind, placebo-controlled trial. Hepatol Res. 2014;44:83-91.

15. Sakaida I, Kawazoe S, Kajimura K, et al. Tolvaptan for improvement of hepatic edema: a phase 3, multicenter, randomized, doubleblind, placebo-controlled trial. Hepatol Res. 2014;44:73-82.

16. Sakaida I, Okita K. Correlation between changes in bodyweight and changes in ascites volume in liver cirrhosis patients with hepatic edema in short-term diuretic therapy. Hepatol Res. 2014;44:735-9.

17. Kasahara A, Kita K, Tomita E, et al. Repeated administration of recombinant human serum albumin caused no serious allergic reactions in patients with liver cirrhosis: a multicenter clinical study. J Gastroenterol. 2008;43:464-72.

18. Moore KP, Wong F, Gines P, et al. The management of ascites in cirrhosis: report on the consensus conference of the International Ascites Club. Hepatology. 2003;38:258-66.

19. Williams JR. The declaration of helsinki and public health. Bull World Health Organ. 2008;86:650-2.

20. Kaur S, Choy CY. Ethical considerations in clinical trials: a critique of the ICH-GCP guideline. Dev World Bioeth. 2014;14: $20-8$.

21. Phakdeekitcharoen B, Boonyawat K. The added-up albumin enhances the diuretic effect of furosemide in patients with hypoalbuminemic chronic kidney disease: a randomized controlled study. BMC Nephrol. 2012. doi:10.1186/1471-2369-13-92.

22. Dharmaraj R, Hari P, Bagga A. Randomized cross-over trial comparing albumin and frusemide infusions in nephrotic syndrome. Pediatr Nephrol. 2009;24:775-82.

23. Péron JM, Bureau C, Gonzalez L, et al. Treatment of hepatorenal syndrome as defined by the international ascites club by albumin and furosemide infusion according to the central venous pressure: a prospective pilot study. Am J Gastroenterol. 2005;100:2702-7.

24. Na KY, Han JS, Kim YS, et al. Does albumin preinfusion potentiate diuretic action of furosemide in patients with nephrotic syndrome? J Korean Med Sci. 2001;16:448-54.

25. Inoue M, Okajima K, Itoh K, et al. Mechanism of furosemide resistance in analbuminemic rats and hypoalbuminemic patients. Kidney Int. 1987;32:198-203.

26. Gentilini P, Casini-Raggi V, Di Fiore G, et al. Albumin improves the response to diuretics in patients with cirrhosis and ascites: results of a randomized, controlled trial. J Hepatol. 1999;30: 639-45.

27. Brater DC. Diuretic therapy. N Engl J Med. 1998;339:387-95.

28. Anderson RJ, Chung HM, Kluge R, et al. Hyponatremia: a prospective analysis of its epidemiology and the pathogenetic role of vasopressin. Ann Intern Med. 1985;102:164-8.

29. Furukawa M, Umehara K, Kashiyama E. Nonclinical pharmacokinetics of a new nonpeptide V2 receptor antagonist, tolvaptan. Cardiovasc Drugs Ther. 2011;25(Suppl 1):S83-9.

30. Hori M. Tolvaptan for heart failure patients with volume overload. Cardiovasc Drugs Ther. 2011;25(Suppl 1):S1-4.

31. Kinugawa K, Sato N, Inomata T, et al. Efficacy and safety of tolvaptan in heart failure patients with volume overload. Circ J. 2014;78:844-52. 